\title{
Iron and Oxidative Status Following Routine Iron Supplementation: Reflection on Pregnancy Outcomes in a Cohort of Women in Kinshasa, DR Congo
}

\author{
Andy Mbangama Muela, Barthélémy Tandu-Umba*, Roger Mbungu Mwimba \\ Department of Obstetrics and Gynecology, University Clinics of Kinshasa, Kinshasa, Congo \\ Email: dymuela@yahoo.fr
}

How to cite this paper: Muela, A.M. Tandu-Umba, B. and Mwimba, R.M. (2019) Iron and Oxidative Status Following Routine Iron Supplementation: Reflection on Pregnancy Outcomes in a Cohort of Women in Kinshasa, DR Congo. Open Journal of Obstetrics and Gynecology, 9, 582-596.

https://doi.org/10.4236/ojog.2019.95057

Received: January 31, 2019

Accepted: May 10, 2019

Published: May 13, 2019

Copyright (C 2019 by author(s) and Scientific Research Publishing Inc. This work is licensed under the Creative Commons Attribution International License (CC BY 4.0).

http://creativecommons.org/licenses/by/4.0/

\begin{abstract}
Background: Anemia during pregnancy is associated with oxidative stress, which might expectedly provoke harmful consequences on maternal and perinatal outcomes. Use of iron in women during pregnancy improves maternal hematologic parameters, while likely to worsen oxidative status. Objectives: Our study thus aimed to assess adverse outcomes on all women having been routinely iron supplemented during pregnancy. Methods: This is a prospective observational cohort of 74 pregnant women with singleton pregnancy whose baseline iron and oxidative status along with variations throughout pregnancy have been recently assessed at university clinics of Kinshasa, DR Congo. Obstetrical adverse outcomes were assessed according to the diagnosis of anemia and oxidative stress considered at recruitment, at 28 - 32 weeks of gestation and at term. For statistical calculations, we used t-test, chi-square test, ANOVAR and regression, the significance being stated at $p<0.05$. Results: Complications significantly associated with anemia at the beginning of pregnancy were acute fetal distress $(\mathrm{OR}=3.9, \mathrm{p}<0.03)$, prematurity $(\mathrm{OR}=$ $7.3, \mathrm{p}<0.007)$, low birth weight or LBW $(\mathrm{OR}=3.4, \mathrm{p}<0.05)$, birth asphyxia $(\mathrm{OR}=15.1, \mathrm{p}<0.002)$ and neonatal hypoglycemia $(\mathrm{OR}=3, \mathrm{p}<0.05)$. When the diagnosis of anemia was considered at 28 - 32 weeks of gestation, significant associations were found with gestational diabetes mellitus or GDM (OR $=3.8, \mathrm{p}<0.05)$, cesarean section $(\mathrm{OR}=4.8, \mathrm{p}<0.003)$, prematurity $(\mathrm{OR}=$ $5.3, \mathrm{p}<0.03)$, birth asphyxia $(\mathrm{OR}=10.9, \mathrm{p}<0.008)$ and neonatal hypoglycemia $(\mathrm{OR}=4.7, \mathrm{p}<0.02)$. At term, the diagnosis of anemia was significantly associated with GDM $(\mathrm{OR}=9.2, \mathrm{p}<0.01)$, premature rupture of membranes or PROM (OR $=2.8, \mathrm{p}<0.05)$, cesarean section $(\mathrm{OR}=6.03, \mathrm{p}<0.01)$, birth asphyxia $(\mathrm{OR}=2.9, \mathrm{p}<0.05)$ and neonatal hypoglycemia $(\mathrm{OR}=3, \mathrm{p}<0.05)$.
\end{abstract}

${ }^{\star}$ We regret to announce the death of Professor Barthelemy Tandu-Umba since March 25, 2019. 
As of oxidative stress diagnosed at recruitment, significant associations were found with PROM $(\mathrm{OR}=9, \mathrm{p}<0.02)$, cesarean section $(\mathrm{OR}=3.7, \mathrm{p}<0.05)$, prematurity $(\mathrm{OR}=6.4, \mathrm{p}<0.02)$, birth asphyxia $(\mathrm{OR}=13.2, \mathrm{p}<0.004)$ and neonatal hypoglycemia $(\mathrm{OR}=2.6, \mathrm{p}<0.05)$. The diagnosis of oxidative stress at 28 - 32 weeks of gestation was found significantly associated with acute fetal distress $(\mathrm{OR}=4.2, \mathrm{p}<0.02)$, cesarean section $(\mathrm{OR}=2.9, \mathrm{p}<0.05)$, LBW $(\mathrm{OR}=9.9, \mathrm{p}<0.002)$, birth asphyxia $(\mathrm{OR}=3.9, \mathrm{p}<0.05)$ and neonatal hypoglycemia $(\mathrm{OR}=3.2, \mathrm{p}<0.04)$. For oxidative stress diagnosed at term, significant associations concerned GDM ( $\mathrm{OR}=11.4, \mathrm{p}<0.006)$, preeclampsia or $\mathrm{PE}(\mathrm{OR}=4.3, \mathrm{p}<0.03)$, acute fetal distress $(\mathrm{OR}=8.3, \mathrm{p}<0.003)$, cesarean section $(\mathrm{OR}=4.3, \mathrm{p}<0.007)$, prematurity $(\mathrm{OR}=6, \mathrm{p}<0.02)$, LBW $(\mathrm{OR}=$ $4.3, \mathrm{p}<0.03)$, birth asphyxia $(\mathrm{OR}=12.4, \mathrm{p}<0.005)$ and neonatal hypoglycemia $(\mathrm{OR}=3.5, \mathrm{p}<0.04)$. Conclusion: Outcomes found significantly associated with oxidative stress seem to overlap those linked to anemic condition. Similarity between complications of anemia and that of oxidative stress is more observed at 28 - 32 weeks of gestation. This strongly suggests that major correction in both anemic and oxidative status should be initiated long before this landmark.

\section{Keywords}

Routine Iron Supplementation, Iron and Oxidative Status, Pregnancy Outcomes, Kinshasa

\section{Introduction}

Maternal iron-deficiency anemia is well known to be associated with high risk of shortening of pregnancy, impairment of fetal growth in terms of low birth weight (LBW) [1] [2], as well as significant decreased Apgar score during the first and the fifth minute [3].

Use of iron in women during pregnancy improves hematologic values [4] [5] and is expected to prevent these adverse outcomes. The beneficial effect has been evidenced as depending on dose of iron, duration of use, and baseline hemoglobin $(\mathrm{Hb})$ concentration before treatment [5] [6].

However, iron supplementation both reverses the weakening of anti-oxidant defenses and increases lipid peroxidation [5] [7]-[12], which has been claimed to increase the generation of free radicals leading to oxidative stress [13] [14] [15] [16].

Consequences associated with damages resulting from stress mediators cover a wide field of manifestations such as gestational diabetes mellitus (GDM), preeclampsia (PE), premature rupture of membranes (PROM) and fetal outcomes such as prematurity, LBW, macrosomia, perinatal morbidity/death, and postpartum infections [17]. So, anemic condition is likely to generate the same consequences as that attributed to pro-oxidant properties of iron used to prevent it [18]. 
Evolution of iron and related oxidative status alongside iron supplementation thus means that beneficial effect of the treatment will vary according to the level of iron-deficiency throughout pregnancy [5]. Therefore, a routine administration may be harmful for women who are or become iron-replete at any period of supplementation. Indeed, possibility of adverse outcomes, along with that of oxidative damage due to iron overload, has been found lower or higher if the diagnosis of anemia is considered at the beginning, mid-pregnancy or later during pregnancy.

The present study thus aimed to assess adverse pregnancy outcomes on all women having been routinely iron supplemented during pregnancy in order to answer the question: can iron supplementation prevent as well as induce maternal and perinatal complications of oxidative stress?

\section{Methods}

The current study is a prospective observational cohort of 74 pregnant women with singleton pregnancy and was approved by the Ethics Committee of the School of Public Health of the University of Kinshasa, DR Congo. It was conducted during 10 months (from September 2017 throughout June 2018), the protocol of which was recently published [19]. The study sample was represented by pregnant women with pregnancy not exceeding 19 weeks (starting point of iron supplementation), without obvious pathology likely to have a negative influence on pregnancy (hypertension, diabetes mellitus, sickle cell disease, HIV, malaria, respiratory insufficiency) and regardless of age and parity. Eligible women were definitely recruited after informed and written consent to participate in the study. Only women reaching fetal viability (28 weeks of gestation) were selected. Irrespective of their biochemical status, all women of this cohort received routine Fe supplementation according to the national policy. Women with anemia (hemoglobin or $\mathrm{Hb}<10 \mathrm{~g} / \mathrm{dl}$ ) took iron tablets for curative purposes $(320 \mathrm{mg} /$ day of ferric ammonium citrate), while the others received preventive iron supplementation (160 mg/day of ferric ammonium citrate). Let us note that these dosages have never been questioned in the country. Iron was associated with $15 \mathrm{mg}$ folate/day. Nutritional iron intake was measured through a 24-hour recall using a questionnaire of common foods, including stuffs and habits likely to impede iron absorption, such as pica/geophagia, tannins and fibers. Supplemental iron intake was determined with a sheet containing details on the duration of the treatment. Additionally to baseline measurements at recruitment ( \pm 15 weeks of gestation) blood sampling and analysis were carried out at 28 - 32 weeks and at term [5] [20]. Biological parameters were: Hb, hematocrit, ferritin, serum iron, transferrin and iron saturation capacity for iron status and superoxide dismutase (SOD), uric acid, oxidized low-density lipoprotein (LDL) and fasting blood glucose for oxidative status.

Throughout confinement adverse obstetrical outcomes were registered in terms of preterm labor and premature delivery ( $<37$ weeks of gestation), $\mathrm{PE}$ (hypertension considered as blood pressure $\geq 140 / 90 \mathrm{mmHg}$ ), GDM (to be di- 
agnosed between weeks 26 and 28 of gestation using a glucose tolerance test according to the "Hyperglycaemia and Adverse Pregnancy Outcomes or HAPO study" [21]), PROM, prematurity (birth between 28 weeks of gestation and 37 weeks), LBW (birth weight $<2500 \mathrm{~g}$ ), stillbirth (defined as death of a fetus after 28 weeks of gestation), perinatal mortality (deaths including stillbirths and neonatal deaths before 7 days of life), birth asphyxia or neonatal distress (defined as an Apgar score $<7$ at the fifth minute) and any other neonatal or postpartum morbidity [19].

Data were registered using Microsoft Excel (Microsoft Corporation, Redmond, WA, USA, 2007) and after transfer to statistical package for social sciences or SPSS (version 18.0; SPSS Inc., Chicago, IL, USA). For statistical calculations, univariate analyses (odds ratios [ORs]) were stratified in a dichotomic way (with or without anemia, with and without oxidative stress, with and without iron/oxidative status variations) to seek significant association with adverse pregnancy outcomes. Multivariate calculations (regression) using various risk factors were aimed at isolating those significantly influencing occurrence of complications in anemic/stressed mothers. Results are given with $95 \%$ confidence intervals (CIs), and $\mathrm{p}<0.05$ was considered significant.

\section{Results}

Women of the study $(n=74)$ were recruited at $15.9 \pm 1.7$ weeks of gestation and were aged $32.2 \pm 5.2$ years. Anemia was diagnosed to 39 women that represented $52.2 \%$ of the study group. Their main characteristics were as follow: $69.2 \%$ of which from low socioeconomic level, mean parity, weight, and body mass index or BMI of $2 \pm 1,70.2 \pm 14.5 \mathrm{Kg}$ and $26 \pm 5.2 \mathrm{Kg} / \mathrm{m}^{2}$, respectively. The proportion of intestinal parasitosis (48.6\%) was significantly higher among anemic women $(61.5 \%$ vs $34.3 \%$; $\mathrm{P}<0.02)$ that had significantly lower alimentary iron intake $(22.8 \pm 4.9$ gr vs $31.4 \pm 9.5$ gr; $\mathrm{P}<0.001)$. No stillbirth was observed.

Maternal and neonatal characteristics at delivery are depicted in Table 1. The mean gestational age was $37.5 \pm 1.9$ weeks and $81.1 \%$ of deliveries occurred at term and in $55.4 \%$ of cases by vaginal delivery. The mean of birth weight was $2978.9 \pm 626.1$ gr. Average Apgar score was $8 \pm 1$ at birth, $9 \pm 1$ at the fifth and 9 \pm 1 at the tenth minute. Table 1 show that $82.4 \%$ of newborns had a good Apgar score at birth, $95.9 \%$ at the fifth and all of them at the tenth minute. There were significantly more newborns with LBW $(28.2 \%$ vs $11.4 \%, \mathrm{p}<0.04)$ and those with a bad Apgar at the 1 st minute $(25.6 \%$ vs $8.6 \%, p<0.04)$ in the group of pregnant women with anemia.

Table 2 present adverse outcomes that were registered in the study sample, namely: GDM, PE, PROM, acute fetal distress, cesarean section, prematurity, LBW, birth asphyxia (neonatal distress) and neonatal hypoglycemia.

The adverse outcomes that were significantly more frequent in the group of pregnant women with anemia compared to those without anemia are (Table 3 and Table 4):

- at the beginning of pregnancy (14 - 19 weeks): acute fetal distress (76.5\% vs 
$23.5 \%, \mathrm{p}<0.03)$, prematurity (85.7\% vs $14.3 \%$, p < 0.01), LBW (75\% vs 25 , p $<0.05)$, birth asphyxia $(92.3 \%$ vs $7.7 \%, \mathrm{p}<0.01)$ and neonatal hypoglycemia (72.2\% vs $27.8 \%, \mathrm{p}<0.05)$;

- $\quad$ at the middle of pregnancy (28 - 32 weeks): GDM ( $81.3 \%$ vs $18.8 \%, \mathrm{p}<0.05)$, PROM (90\% vs $10 \%, \mathrm{p}<0.04)$, caesarean section $(78.8 \%$ vs $21.2 \%, \mathrm{p}<0.01)$, prematurity $(85.7 \%$ vs $14.3 \%$, p < 0.03), birth asphyxia $(92.3 \%$ vs $7.7 \%$, p < $0.01)$ and neonatal hypoglycemia ( $83.3 \%$ vs $16.7 \%, \mathrm{p}<0.02)$;

- and at the end of pregnancy: GDM (87.5\% vs. $12.5 \%, \mathrm{p}<0.01)$, PE (75\% vs. $25 \%, \mathrm{p}<0.05)$, caesarean section $(75.8 \%$ vs $24.2 \%, \mathrm{p}<0.001)$ and neonatal hypoglycemia $(72.2 \%$ vs $27.8 \%$, p $<0.05)$.

Using non adjusted OR, the next step of our calculations (Tables 5-7) questioned the effect of anemia and oxidative stress on each of these outcomes.

Table 1. Maternal and neonatal characteristics at delivery.

\begin{tabular}{|c|c|c|c|c|}
\hline Characteristics & Overall group & $\begin{array}{l}\text { Anemic women } \\
\quad(\mathrm{n}=39)\end{array}$ & $\begin{array}{l}\text { Non anemic women } \\
\qquad(\mathrm{n}=35)\end{array}$ & $\mathrm{p}$ \\
\hline Age (yrs) & $32.2 \pm 5.2$ & $32.3 \pm 4.7$ & $32.03 \pm 5.7$ & 0.82 \\
\hline Parity & $2 \pm 1$ & $2 \pm 1$ & $2 \pm 1$ & 0.171 \\
\hline Gravidity & $3 \pm 1$ & $3 \pm 1$ & $3 \pm 1$ & 0.247 \\
\hline \multicolumn{5}{|l|}{ Gestational age at delivery } \\
\hline Mean & $37.5 \pm 1.9$ weeks & $37.4 \pm 1.9$ weeks & $37.6 \pm 1.8$ weeks & 0.586 \\
\hline$<37$ weeks & $14(18.9 \%)$ & $10(25.6 \%)$ & $4(11.4 \%)$ & 0.103 \\
\hline$\geq 37$ weeks & $60(81.1 \%)$ & $29(74.4 \%)$ & $31(88.6 \%)$ & \\
\hline \multicolumn{5}{|l|}{ Mode of delivery } \\
\hline Vaginal delivery & $41(55.4 \%)$ & $19(48.7 \%)$ & $22(62.9 \%)$ & 0.162 \\
\hline Cesarean section & $33(44.6 \%)$ & $20(51.3 \%)$ & $13(37.1 \%)$ & \\
\hline \multicolumn{5}{|l|}{ Birth weight } \\
\hline Mean & $2978.9 \pm 626.1 \mathrm{gr}$ & $2860 \pm 610.7 \mathrm{gr}$ & $3111.4 \pm 624.9 \mathrm{gr}$ & 0.085 \\
\hline LBW (<2500 gr) & $15(20.3 \%)$ & $11(28.2 \%)$ & $4(11.4 \%)$ & \\
\hline Normal birth weight $(2500-<4000$ gr $)$ & $52(70.3 \%)$ & $26(66.7 \%)$ & $26(74.3 \%)$ & $<0.04$ \\
\hline Macrosomia ( $\geq 4000$ gr) & $7(9.5 \%)$ & $2(5.1 \%)$ & $5(14.3 \%)$ & \\
\hline \multicolumn{5}{|l|}{ Apgar score at the first minute } \\
\hline Mean & $8 \pm 1$ & $7 \pm 1$ & $8 \pm 1$ & $<0.04$ \\
\hline$<7$ & $13(17.6 \%)$ & $10(25.6 \%)$ & $3(8.6 \%)$ & $<0.05$ \\
\hline$\geq 7$ & $61(82.4 \%)$ & $29(74.4 \%)$ & $32(91.4 \%)$ & \\
\hline \multicolumn{5}{|l|}{ Apgar score at the fifth minute } \\
\hline Mean & $9 \pm 1$ & $8 \pm 1$ & $9 \pm 1$ & 0.176 \\
\hline$<7$ & $3(4.1 \%)$ & $1(2.6 \%)$ & $2(5.7 \%)$ & 0.459 \\
\hline$\geq 7$ & $71(95.9 \%)$ & $38(97.4 \%)$ & $33(94.3 \%)$ & \\
\hline \multicolumn{5}{|l|}{ Apgar score at the tenth minute } \\
\hline Mean & $9 \pm 1$ & $9 \pm 1$ & $9 \pm 1$ & $<0.04$ \\
\hline$<7$ & - & - & - & - \\
\hline$\geq 7$ & $74(100 \%)$ & $39(100 \%)$ & $35(100 \%)$ & \\
\hline
\end{tabular}


Table 2. Adverse pregnancy outcomes of the study sample based on anemia $(\mathrm{Hb}<10 \mathrm{~g} / \mathrm{dl})$.

\begin{tabular}{|c|c|c|c|c|c|c|c|c|c|c|c|c|c|c|c|c|c|}
\hline \multirow[b]{3}{*}{ Outcomes } & \multicolumn{7}{|c|}{14 - 19 weeks } & \multicolumn{5}{|c|}{$28-32$ weeks } & \multicolumn{5}{|c|}{ At term } \\
\hline & \multicolumn{2}{|c|}{ Total } & \multicolumn{2}{|c|}{ Anemia } & \multicolumn{3}{|c|}{ Normal } & \multicolumn{2}{|c|}{ Anemia } & \multicolumn{2}{|c|}{ Normal } & \multicolumn{3}{|c|}{ Anemia } & \multicolumn{2}{|c|}{ Normal } & \multirow[b]{2}{*}{$\mathrm{p}$} \\
\hline & $(n=74)$ & $\%$ & $(\mathrm{n}=39)$ & $\%$ & $(\mathrm{n}=35)$ & $\%$ & $\mathrm{p}$ & $(\mathrm{n}=44)$ & $\%$ & $(\mathrm{n}=30)$ & $\%$ & $\mathrm{p}$ & $(n=39)$ & $\%$ & $(n=35)$ & $\%$ & \\
\hline GDM & 16 & 21.6 & 8 & 50 & 8 & 50 & 0.514 & 13 & 81.2 & 3 & 18.8 & $<0.05$ & 14 & 87.5 & 2 & 12.5 & $<0.01$ \\
\hline $\mathrm{PE}$ & 16 & 21.6 & 9 & 56.2 & 7 & 43.8 & 0.486 & 11 & 68.8 & 5 & 31.2 & 0.288 & 12 & 75 & 4 & 25 & $<0.05$ \\
\hline PROM & 10 & 13.5 & 7 & 70 & 3 & 30 & 0.202 & 9 & 90 & 1 & 10 & $<0.04$ & 6 & 60 & 4 & 40 & 0.440 \\
\hline $\begin{array}{l}\text { Acute fetal } \\
\text { distress }\end{array}$ & 17 & 23 & 13 & 76.5 & 4 & 23.5 & $<0.03$ & 12 & 70.6 & 5 & 29.4 & 0.218 & 10 & 58.8 & 7 & 41.2 & 0.384 \\
\hline $\begin{array}{c}\text { Cesarean } \\
\text { section }\end{array}$ & 33 & 44.6 & 20 & 60.6 & 13 & 39.4 & 0.162 & 26 & 78.8 & 7 & 21.2 & $<0.01$ & 25 & 75.8 & 8 & 24.2 & $<0.001$ \\
\hline Prematurity & 14 & 18.9 & 12 & 85.7 & 2 & 14.3 & $<0.01$ & 12 & 85.7 & 2 & 14.3 & $<0.03$ & 10 & 71.4 & 4 & 28.6 & 0.103 \\
\hline LBW & 16 & 21.6 & 12 & 75 & 4 & 25 & $<0.05$ & 11 & 68.8 & 5 & 31.2 & 0.288 & 6 & 37.5 & 10 & 62.5 & 0.137 \\
\hline Birth asphyxia & 13 & 17.6 & 12 & 92.3 & 1 & 7.7 & $<0.01$ & 12 & 92.3 & 1 & 7.7 & $<0.01$ & 8 & 61.5 & 5 & 38.5 & 0.348 \\
\hline $\begin{array}{c}\text { Neonatal } \\
\text { hypoglycemia }\end{array}$ & 18 & 24.3 & 13 & 72.2 & 5 & 27.8 & $<0.05$ & 15 & 83.3 & 3 & 16.7 & $<0.02$ & 13 & 72.2 & 5 & 27.8 & $<0.05$ \\
\hline
\end{tabular}

Table 3. Adverse pregnancy outcomes of the study sample based on SOD (normal 785 - 1570 UI/L).

\begin{tabular}{|c|c|c|c|c|c|c|c|c|c|c|c|c|c|c|c|}
\hline \multirow[b]{3}{*}{ Outcomes } & \multicolumn{5}{|c|}{14 - 19 weeks } & \multicolumn{5}{|c|}{28 - 32 weeks } & \multicolumn{5}{|c|}{ At term } \\
\hline & \multicolumn{2}{|c|}{$\begin{array}{c}\text { Pathologic } \\
\text { SOD }\end{array}$} & \multicolumn{2}{|c|}{ Normal SOD } & & \multicolumn{2}{|c|}{$\begin{array}{c}\text { Pathologic } \\
\text { SOD }\end{array}$} & \multicolumn{2}{|c|}{ Normal SOD } & \multirow[b]{2}{*}{$\mathrm{p}$} & \multicolumn{2}{|c|}{ Pathologic SOD } & \multicolumn{2}{|c|}{ Normal SOD } & \multirow[b]{2}{*}{$\mathrm{p}$} \\
\hline & $(n=60)$ & $\%$ & $(n=14)$ & $\%$ & & $(n=52)$ & $\%$ & $(n=22)$ & $\%$ & & $(n=48)$ & $\%$ & $(n=26)$ & $\%$ & \\
\hline GDM & 14 & 87.5 & 2 & 12.5 & 0.368 & 13 & 81.2 & 3 & 18.8 & 0.222 & 15 & 93.8 & 1 & 6.2 & $<0.01$ \\
\hline PE & 13 & 81.2 & 3 & 18.8 & 0.647 & 12 & 75 & 4 & 25 & 0.447 & 11 & 68.8 & 5 & 31.2 & 0.478 \\
\hline PROM & 8 & 80 & 2 & 20 & 0.607 & 5 & 50 & 5 & 50 & 0.129 & 7 & 70 & 3 & 30 & 0.507 \\
\hline Acute fetal distress & 15 & 88.2 & 2 & 11.8 & 0.319 & 13 & 76.5 & 4 & 23.5 & 0.377 & 12 & 70.6 & 6 & 29.4 & 0.398 \\
\hline Cesarean section & 30 & 90.9 & 3 & 9.1 & $<0.05$ & 27 & 81.8 & 6 & 18.2 & $<0.05$ & 27 & 81.8 & 6 & 18.2 & $<0.01$ \\
\hline Prematurity & 12 & 85.7 & 2 & 14.3 & 0.476 & 9 & 64.3 & 5 & 35.7 & 0.403 & 8 & 57.1 & 6 & 42.9 & 0.353 \\
\hline LBW & 15 & 93.8 & 1 & 6.2 & 0.133 & 11 & 68.8 & 5 & 31.2 & 0.553 & 9 & 56.2 & 7 & 43.8 & 0.298 \\
\hline Birth asphyxia & 12 & 92.3 & 1 & 7.7 & 0.237 & 9 & 69.2 & 4 & 30.8 & 0.584 & 8 & 61.5 & 5 & 38.5 & 0.509 \\
\hline Neonatal hypoglycemia & 15 & 83.3 & 3 & 16.7 & 0.541 & 14 & 77.8 & 4 & 22.2 & 0.313 & 12 & 66.7 & 6 & 33.3 & 0.545 \\
\hline
\end{tabular}

Table 4. Adverse pregnancy outcomes of the study sample based on oxidized LDL (normal < 600 UI/L).

\begin{tabular}{|c|c|c|c|c|c|c|c|c|c|c|c|c|c|c|c|}
\hline \multirow[b]{3}{*}{ Outcomes } & \multicolumn{5}{|c|}{14 - 19 weeks } & \multicolumn{5}{|c|}{28 - 32 weeks } & \multicolumn{5}{|c|}{ At term } \\
\hline & \multicolumn{2}{|c|}{$\begin{array}{c}\text { Pathologic } \\
\text { oxidized LDL }\end{array}$} & \multicolumn{2}{|c|}{$\begin{array}{c}\text { Normal } \\
\text { oxidized LDL }\end{array}$} & \multirow[b]{2}{*}{$\mathrm{p}$} & \multicolumn{2}{|c|}{$\begin{array}{c}\text { Pathologic } \\
\text { oxidized LDL }\end{array}$} & \multicolumn{2}{|c|}{$\begin{array}{c}\text { Normal } \\
\text { oxidized LDL }\end{array}$} & \multirow[b]{2}{*}{$\mathrm{p}$} & \multicolumn{2}{|c|}{$\begin{array}{c}\text { Pathologic } \\
\text { oxidized LDL }\end{array}$} & \multicolumn{2}{|c|}{$\begin{array}{c}\text { Normal } \\
\text { oxidized LDL }\end{array}$} & \multirow[b]{2}{*}{$\mathrm{p}$} \\
\hline & $(n=41)$ & $\%$ & $(n=33)$ & $\%$ & & $(\mathrm{n}=38)$ & $\%$ & $(n=36)$ & $\%$ & & $(n=42)$ & $\%$ & $(\mathrm{n}=32)$ & $\%$ & \\
\hline GDM & 9 & 56.3 & 7 & 43.8 & 0.584 & 6 & 37.5 & 10 & 62.5 & 0.166 & 8 & 50 & 8 & 50 & 0.368 \\
\hline $\mathrm{PE}$ & 8 & 50 & 8 & 50 & 0.416 & 11 & 68.8 & 5 & 31.3 & 0.098 & 13 & 81.2 & 3 & 18.8 & $<0.03$ \\
\hline PROM & 9 & 90 & 1 & 10 & $<0.02$ & 3 & 30 & 7 & 70 & 0.133 & 7 & 70 & 3 & 30 & 0.290 \\
\hline
\end{tabular}




\section{Continued}

\begin{tabular}{|c|c|c|c|c|c|c|c|c|c|c|c|c|c|c|c|}
\hline Acute fetal distress & 12 & 70.6 & 5 & 29.4 & 0.123 & 13 & 76.5 & 4 & 23.5 & $<0.02$ & 15 & 88.2 & 2 & 11.8 & $<0.003$ \\
\hline Cesarean section & 20 & 60.6 & 13 & 39.4 & 0.284 & 18 & 54.5 & 15 & 45.5 & 0.398 & 21 & 63.6 & 12 & 36.4 & 0.202 \\
\hline Prematurity & 12 & 85.7 & 2 & 14.3 & $<0.02$ & 8 & 57.1 & 6 & 42.9 & 0.428 & 12 & 85.7 & 2 & 14.3 & $<0.02$ \\
\hline LBW & 10 & 62.5 & 6 & 37.5 & 0.362 & 14 & 87.5 & 2 & 12.5 & $<0.002$ & 13 & 81.2 & 3 & 18.8 & $<0.03$ \\
\hline Neonatal hypoglycemia & 13 & 72.2 & 6 & 27.8 & $<0.05$ & 13 & 72.2 & 5 & 27.8 & $<0.04$ & 14 & 77.8 & 4 & 22.2 & $<0.04$ \\
\hline
\end{tabular}

Table 5. Adverse maternal and perinatal outcomes associated with anemia ( $<10 \mathrm{~g} / \mathrm{dl})$.

\begin{tabular}{ccccccccccc}
\hline Outcomes & \multicolumn{3}{c}{$14-19$ weeks } & \multicolumn{2}{c}{$28-32$ weeks } & \multicolumn{3}{c}{ At term } \\
\hline & $\mathrm{p}$ & OR & CI $95 \%$ & $\mathrm{p}$ & OR & CI 95\% & p & OR & CI 95\% \\
\hline GDM & 0.514 & 1.1 & $0.5-2.7$ & $<0.05$ & 3.8 & $1.1-14.7$ & $<0.01$ & 9.2 & $1.9-44.4$ \\
PE & 0.486 & 1.2 & $0.4-3.7$ & 0.288 & 1.7 & $0.5-5.4$ & $<0.05$ & 3.4 & $1.1-11.9$ \\
PROM & 0.202 & 2.3 & $0.6-9.8$ & $<0.04$ & 7.5 & $1.9-62.4$ & 0.440 & 1.4 & $0.4-5.5$ \\
Acute fetal distress & $<0.03$ & 3.9 & $1.1-13.3$ & 0.218 & 1.9 & $0.6-6.02$ & 0.384 & 1.4 & $0.5-4.1$ \\
Cesarean section & 0.162 & 1.8 & $0.7-4.5$ & $<0.003$ & 4.8 & $1.7-13.4$ & $<0.01$ & 6.03 & $2.2-16.8$ \\
Prematurity & $<0.007$ & 7.3 & $1.5-35.6$ & $<0.03$ & 5.3 & $1.1-25.5$ & 0.103 & 2.7 & $0.8-9.5$ \\
LBW & $<0.05$ & 3.4 & $1.1-11.9$ & 0.288 & 1.7 & $0.5-5.4$ & 0.137 & 1.9 & $0.8-4.6$ \\
Birth asphyxia & $<0.002$ & 15.1 & $1.9-123.6$ & $<0.008$ & 10.9 & $1.3-88.9$ & 0.348 & 1.6 & $0.5-5.3$ \\
Neonatal hypoglycemia & $<0.05$ & 3 & $1.1-9.6$ & $<0.02$ & 4.7 & $1.2-17.9$ & $<0.05$ & 3 & $1.01-9.6$ \\
\hline
\end{tabular}

Table 6. Adverse outcomes associated with pathologic iron status.

\begin{tabular}{|c|c|c|c|c|c|c|c|c|c|c|c|c|c|c|c|c|c|c|}
\hline \multirow{3}{*}{ Outcomes } & \multicolumn{9}{|c|}{ FERRITIN $(<15$ ou $>150 \mathrm{ng} / \mathrm{mL})$} & \multicolumn{9}{|c|}{ Serum iron $(>31 \mu \mathrm{g} / \mathrm{dl})$} \\
\hline & \multicolumn{3}{|c|}{$14-19$ weeks } & \multicolumn{3}{|c|}{28 - 32 weeks } & \multicolumn{3}{|c|}{ At term } & \multicolumn{3}{|c|}{14 - 19 weeks } & \multicolumn{3}{|c|}{28 - 32 weeks } & \multicolumn{3}{|c|}{ At term } \\
\hline & $\mathrm{p}$ & OR & CI $95 \%$ & $\mathrm{p}$ & OR & CI $95 \%$ & $\mathrm{p}$ & OR & CI $95 \%$ & $\mathrm{p}$ & OR & CI $95 \%$ & $\mathrm{p}$ & OR & CI $95 \%$ & $\mathrm{p}$ & OR & CI $95 \%$ \\
\hline GDM & 0.611 & 1 & $0.3-3.03$ & 0.071 & 2.7 & $0.9-8.6$ & 0.147 & 2.2 & $0.7-7.1$ & 0.313 & 2.02 & $0.4-10.1$ & 0.630 & 1.1 & $0.2-5.9$ & 0.527 & 1.7 & $0.2-15.5$ \\
\hline PROM & $<0.05$ & 4.8 & $1.1-24.5$ & 0.209 & 2.2 & $0.6-8.5$ & $<0.05$ & 2.8 & $1.1-9.8$ & 0.674 & 1.02 & $0.2-5.4$ & 0.594 & 1.5 & $0.2-13.1$ & 0.655 & 1.1 & $0.2-7.2$ \\
\hline $\begin{array}{l}\text { Acute fetal } \\
\text { distress }\end{array}$ & 0.134 & 2.2 & $0.7-6.7$ & 0.116 & 2.3 & $0.8-6.9$ & 0.433 & 1.3 & $0.4-3.9$ & $<0.005$ & 3.5 & $1.6-7.5$ & 0.414 & 1.4 & $0.5-3.9$ & 0.194 & 2.1 & $0.8-5.4$ \\
\hline $\begin{array}{l}\text { Cesarean } \\
\text { section }\end{array}$ & 0.500 & 1.1 & $0.5-2.8$ & 0.146 & 1.8 & $0.7-4.7$ & 0.106 & 2.03 & $0.8-5.2$ & 0.317 & 1.3 & $0.7-2.2$ & 0.515 & 1.2 & $0.3-4.8$ & $<0.03$ & 2.1 & $1.4-3.2$ \\
\hline Prematurity & $<0.04$ & 3.1 & $1.1-10.8$ & 0.392 & 1.4 & $1.4-4.5$ & 0.482 & 1.2 & $0.5-3.02$ & 0.301 & 1.6 & $0.6-4.3$ & 0.393 & 2.3 & $0.3-19.8$ & 0.396 & 1.6 & $0.4-5.7$ \\
\hline LBW & $<0.03$ & 3.9 & $1.1-13.8$ & 0.368 & 1.4 & $0.5-4.3$ & 0.147 & 2.2 & $0.7-7.1$ & $<0.05$ & 2.4 & $1.02-5.5$ & 0.370 & 1.5 & $0.5-4.3$ & 0.473 & 1.4 & $0.4-4.8$ \\
\hline Birth asphyxia & $<0.008$ & 7.4 & $1.5-36.3$ & $<0.04$ & 3.7 & $1.03-13.5$ & 0.388 & 1.5 & $0.4-4.9$ & $<0.004$ & 4.6 & $1.8-11.7$ & $<0.05$ & 2.8 & $1.1-7.5$ & $<0.05$ & 2.9 & $1.03-8.03$ \\
\hline $\begin{array}{c}\text { Neonatal } \\
\text { hypoglycemia }\end{array}$ & $<0.03$ & 3.5 & $1.1-11.1$ & 0.174 & 1.9 & $0.7-5.7$ & 0.551 & 1.1 & $0.4-3.2$ & $<0.04$ & 2.5 & $1.2-5.4$ & 0.542 & 1.3 & $0.3-6.9$ & 0.222 & 1.9 & $0.7-5.02$ \\
\hline
\end{tabular}


Table 7. Adverse outcomes associated with oxidative stress.

\begin{tabular}{|c|c|c|c|c|c|c|c|c|c|c|c|c|c|c|c|c|c|c|}
\hline \multirow[b]{2}{*}{ Outcomes } & \multicolumn{9}{|c|}{ SOD $(<785$ ou $>1570$ UI/L) } & \multicolumn{9}{|c|}{ Oxidized LDL (>600 UI/L) } \\
\hline & \multicolumn{3}{|c|}{$14-19$ weeks } & \multicolumn{3}{|c|}{$28-32$ weeks } & \multicolumn{3}{|c|}{ At term } & \multicolumn{3}{|c|}{$14-19$ weeks } & \multicolumn{3}{|c|}{28 - 32 weeks } & \multicolumn{3}{|c|}{ At term } \\
\hline GDM & 0.368 & 1.8 & $0.4-9.2$ & 0.222 & 2.1 & $0.5-8.3$ & $<0.006$ & 11.4 & $1.4-91.9$ & 0.584 & 1.1 & $0.3-3.2$ & 0.166 & 1.8 & $0.7-4.3$ & 0.368 & 1.3 & $0.6-3.1$ \\
\hline $\mathrm{PE}$ & 0.647 & 1.01 & $0.3-4.2$ & 0.447 & 1.4 & $0.4-4.8$ & 0.478 & 1.3 & $0.4-4.1$ & 0.416 & 1.2 & $0.5-2.9$ & 0.098 & 2.5 & $0.8-8.2$ & $<0.03$ & 4.3 & $1.1-16.8$ \\
\hline PROM & 0.607 & 1.1 & $0.3-4.5$ & 0.129 & 2.4 & $0.8-7.4$ & 0.507 & 1.3 & $0.3-5.6$ & $<0.02$ & 9 & $1.1-75.2$ & 0.133 & 2.5 & $0.7-8.8$ & 0.290 & 1.9 & $0.5-8.2$ \\
\hline $\begin{array}{l}\text { Acute fetal } \\
\text { distress }\end{array}$ & 0.319 & 2 & $0.4-9.9$ & 0.377 & 1.5 & $0.4-5.3$ & 0.398 & 1.5 & $0.4-4.5$ & 0.123 & 2.3 & $0.7-7.4$ & $<0.02$ & 4.2 & $1.2-14.3$ & $<0.003$ & 8.3 & $1.7-39.8$ \\
\hline $\begin{array}{l}\text { Cesarean } \\
\text { section }\end{array}$ & $<0.05$ & 3.7 & $1.1-14.5$ & $<0.05$ & 2.9 & $1.1-8.5$ & $<0.007$ & 4.3 & $1.5-12.6$ & 0.284 & 1.5 & $0.6-3.7$ & 0.398 & 1.3 & $0.5-3.2$ & 0.202 & 1.7 & $0.7-4.3$ \\
\hline LBW & 0.133 & 4.3 & $0.5-35.9$ & 0.553 & 1.1 & $0.4-2.7$ & 0.298 & 1.4 & $0.6-3.4$ & 0.362 & 1.5 & $0.5-4.5$ & $<0.002$ & 9.9 & $2.1-47.7$ & $<0.03$ & 4.3 & $1.1-16.8$ \\
\hline Birth asphyxia & 0.237 & 3.3 & $0.4-27.4$ & 0.584 & 1.1 & $0.4-3.1$ & 0.509 & 1.2 & $0.4-3.2$ & $<0.004$ & 13.2 & $1.6-108.2$ & $<0.05$ & 3.9 & $1.1-15.7$ & $<0.005$ & 12.4 & $1.5-101.4$ \\
\hline $\begin{array}{c}\text { Neonatal } \\
\text { hypoglycemia }\end{array}$ & 0.541 & 1.2 & $0.3-4.9$ & 0.313 & 1.7 & $0.5-5.8$ & 0.545 & 1.1 & $0.4-3.4$ & $<0.05$ & 2.6 & $1.01-8.3$ & $<0.04$ & 3.2 & $1.01-10.3$ & $<0.04$ & 3.5 & $1.03-11.9$ \\
\hline
\end{tabular}

Complications significantly associated with anemia at the beginning of pregnancy were acute fetal distress ( $\mathrm{OR}=3.9$; CI 95\%: $1.1-13.3 ; \mathrm{p}<0.03$; $\mathrm{OR}=3.5$; CI 95\%: $1.6-7.5 ; \mathrm{p}<0.005$ based on serum iron), prematurity (OR $=7.3$; CI 95\%: 1.5 - 35.6; $\mathrm{p}<0.007$; OR = 3.1; CI 95\%: $1.1-10.8$; $\mathrm{p}<0.04$ based on ferritin), LBW (OR = 3.4; CI 95\%: $1.1-11.9 ; \mathrm{p}<0.05 ; \mathrm{OR}=3.9$; CI 95\%: $1.1-13.8 ; \mathrm{p}$ $<0.03$ based on ferritin; OR = 2.4; CI 95\%: $1.02-5.5 ; \mathrm{p}<0.05$ based on serum iron), birth asphyxia or neonatal distress (OR $=15.1$; CI 95\%: $1.9-123.6$; $\mathrm{p}<$ 0.002; $\mathrm{OR}=7.4$; CI 95\%: $1.5-36.3 ; \mathrm{p}<0.008$ based on ferritin; $\mathrm{OR}=4.6$; $\mathrm{CI}$ 95\%: 1.8 - 11.7; $\mathrm{p}<0.004$ based on serum iron) and neonatal hypoglycemia (OR = 3; CI 95\%: $1.1-9.6 ; \mathrm{p}<0.05 ; \mathrm{OR}=3.5$; CI 95\%: $1.1-11.1 ; \mathrm{p}<0.03$ based on ferritin; OR = 2.5; CI 95\%: $1.2-5.4 ; \mathrm{p}<0.04$ based on serum iron).

When the diagnosis of anemia was considered at 28 - 32 weeks of gestation, significant associations were found with Gestational diabetes ( $\mathrm{OR}=3.8$; CI 95\%: 1.1 - 14.7; $\mathrm{p}<0.05)$, PROM (OR = 7.5; CI 95\%: 1.9 - 62.4; $\mathrm{p}<0.04)$, cesarean section ( $\mathrm{OR}=4.8$; CI 95\%: 1.7 - 13.4; $\mathrm{p}<0.003)$, prematurity ( $\mathrm{OR}=5.3$; CI 95\%: 1.1 - 25.5; $\mathrm{p}<0.03)$, birth asphyxia (OR $=10.9$; CI 95\%: $1.3-88.9 ; \mathrm{p}<0.008$; OR $=3.7$; CI 95\%: $1.03-13.5 ; \mathrm{p}<0.04$ based on ferritin; $\mathrm{OR}=2.8$; CI 95\%: $1.1-7.5$; $\mathrm{p}<0.05$ based on serum iron) and neonatal hypoglycemia (OR $=4.7$; CI 95\%: 1.2 - 17.9; $\mathrm{p}<0.02)$.

At term, the diagnosis of anemia was significantly associated with GDM (OR = 9.2; CI 95\%: 1.9 - 44.4; $\mathrm{p}<0.01)$, PE (OR = 3.4; CI 95\%: $1.1-11.9 ; \mathrm{p}<0.05)$, PROM (OR = 2.8; CI 95\%: $1.1-9.8 ; \mathrm{p}<0.05$ based on ferritin), cesarean section $(\mathrm{OR}=6.03$; CI 95\%: $2.2-16.8 ; \mathrm{p}<0.01 ; \mathrm{OR}=2.1$; CI 95\%: $1.4-3.2 ; \mathrm{p}<0.03$ based on serum iron), birth asphyxia (OR = 2.9; CI 95\%: $1.03-8.03$; $\mathrm{p}<0.05$ based on serum iron) and neonatal hypoglycemia (OR $=3$; CI 95\%: $1.01-9.6 ; \mathrm{p}$ $<0.05)$. 
As of oxidative stress diagnosed at recruitment, significant associations were found with PROM (OR = 9; CI 95\%: 1.1 - 75.2; $\mathrm{p}<0.02$ based on oxidized LDL), cesarean section (OR = 3.7; CI 95\%: 1.1 - 14.5; $\mathrm{p}<0.05$ based on SOD), prematurity $(\mathrm{OR}=6.4$; CI 95\%: $1.3-31.2 ; \mathrm{p}<0.02$ based on oxidized LDL), birth asphyxia (OR = 13.2; CI 95\%: $1.6-108.2$; p < 0.004 based on oxidized LDL) and neonatal hypoglycemia $(\mathrm{OR}=2.6$; CI 95\%: $1.01-8.3$; $\mathrm{p}<0.05$ based on oxidized LDL).

The diagnosis of oxidative stress at 28 - 32 weeks of gestation (based on oxidized LDL) was found significantly associated with acute fetal distress $(\mathrm{OR}=4.2$; CI 95\%: 1.2 - 14.3; p < 0.02), cesarean section (OR = 2.9; CI 95\%: $1.1-8.5$; $\mathrm{p}<$ 0.05 based on SOD), LBW (OR = 9.9; CI 95\%: 2.1 - 47.7; p < 0.002), birth asphyxia (OR = 3.9; CI 95\%: $1.1-15.7$; $<<0.05)$ and neonatal hypoglycemia (OR = 3.2; CI 95\%: $1.01-10.3 ; \mathrm{p}<0.04)$.

For oxidative stress diagnosed at term, significant associations concerned GDM (OR = 11.4; CI 95\%: $1.4-91.9 ; \mathrm{p}<0.006$ as of SOD), $\mathrm{PE}(\mathrm{OR}=4.3$; $\mathrm{CI}$ 95\%: 1.1 - 16.8; $\mathrm{p}<0.03$ as of oxidized LDL), acute fetal distress (OR = 8.3; CI 95\%: $1.7-39.8 ; \mathrm{p}<0.003$ as of oxidized $\mathrm{LDL})$, cesarean section $(\mathrm{OR}=4.3$; $\mathrm{CI}$ 95\%: 1.5 - 12.6; $\mathrm{p}<0.007$ as of SOD), prematurity (OR = 6; CI 95\%: $1.2-29.1 ; \mathrm{p}$ $<0.02$ as of oxidized LDL), LBW (OR = 4.3; CI 95\%: $1.1-16.8$; p $<0.03$ as of oxidized LDL), birth asphyxia (OR = 12.4; CI 95\%: $1.5-101.4 ; \mathrm{p}<0.005$ as of oxidized LDL) and neonatal hypoglycemia (OR = 3.5; CI 95\%: 1.03 - 11.9; $\mathrm{p}<$ 0.04 as of oxidized LDL).

Since various pre-pregnancy and pregnancy risk factors and factors related to iron absorption are likely to influence the occurrence of these outcomes, we registered them. Those significantly associated with adverse outcomes served to base adjustment analyses (adjusted OR) of associations.

GDM was significantly associated with intestinal parasitosis $(\mathrm{OR}=3.8$; CI 95\%: 1.1 - 14.7; $\mathrm{p}<0.05)$, geophagia (OR = 4.04; CI 95\%: $1.3-12.8 ; \mathrm{p}<0.02)$ and with hypertension in the family $(\mathrm{OR}=2.7$; CI 95\%: $1.1-7.6 ; \mathrm{p}<0.05)$. PE was significantly associated with intestinal parasitosis (OR = 3.8; CI 95\%: 1.1 - 14.7; p $<0.05)$. Acute fetal distress was significantly associated with antecedent of diabetes in the family (OR $=5.5$; CI 95\%: $1.1-38.9$; $\mathrm{p}<0.03)$. Cesarean section was significantly associated with intestinal parasitosis (OR $=4.8$; CI 95\%: 1.7 13.4; $\mathrm{p}<0.003)$, geophagia ( $\mathrm{OR}=4.6$; CI 95\%: $1.6-13.2 ; \mathrm{p}<0.005)$ and consumption of tannins and fibers (OR = 5.6; CI 95\%: $1.9-15.8 ; \mathrm{p}<0.002)$. Prematurity, was significantly associated with geophagia $(\mathrm{OR}=4$; CI 95\%: $1.2-13.4$; $\mathrm{p}$ $<0.03$ ) and consumption of tannins and fibers (OR = 4.2; CI 95\%: $1.2-14.3$; $\mathrm{p}<$ 0.03). Birth asphyxia (neonatal distress) was significantly associated with antecedent of precocious abortion (OR $=4.4$; CI9\%: $1.2-15.8$; $\mathrm{p}<0.03)$, antecedent of macrosomia (OR = 8.9; CI9\%: $1.3-59.8$; $\mathrm{p}<0.04)$ and consumption of tannins and fibers (OR = 3.5; CI9\%: $1.02-12.2$; $\mathrm{p}<0.05)$.

Subsequently, adjusted OR showed significant links between GDM and SOD at the term $(\mathrm{OR}=5.6$; CI 95\%: $1.01-52.6 ; \mathrm{p}<0.05), \mathrm{PE}$ and Oxidized LDL at the 
term $(\mathrm{OR}=3.9$; CI 95\%: $1.1-15.8 ; \mathrm{p}<0.05)$, cesarean section and serum iron $(\mathrm{OR}=0.03$; CI 95\%: $0.002-0.6 ; \mathrm{p}<0.03), \mathrm{LBW}$ and Oxidized LDL at $28-32$ weeks (OR $=8.2$; CI 95\%: $1.3-52.7$; $\mathrm{p}<0.03$ ) and acute fetal distress and Oxidized LDL at term $(\mathrm{OR}=10.9$; CI 95\%: $1.01-133.2 ; \mathrm{p}<0.05)$.

\section{Discussion}

Our study aimed to assess adverse pregnancy outcomes on all women having been routinely iron supplemented during pregnancy. So, the pending question might be: in what proportion can iron supplementation prevent as well as induce maternal and perinatal complications of oxidative stress?

Even though abundant literature has been claiming anemia strongly associated with adverse outcomes [17] [22] [23] [24], very few studies deal with complications other than prematurity, LBW, PE and GDM. So, our study is among the first to depict such a wide range including GDM, PE, PROM, cesarean section, prematurity, LBW, acute fetal distress, birth asphyxia and neonatal hypoglycemia.

Complications significantly associated with anemia at the beginning of pregnancy were prematurity, LBW, neonatal hypoglycemia and birth asphyxia. When the diagnosis of anemia was considered at 28 - 32 weeks of gestation, meaning absence of correction by iron supplementation, there were quite similar complications: prematurity, GDM, PROM, cesarean section and birth asphyxia. Persistence of anemia till the term worsens the neonatal status, which widens the field of complications with neonatal hypoglycemia. Our findings are in accordance with the fact that maternal anemia actually acts in conjunction with various risk factors in raising the odds of maternal and perinatal morbidity/mortality [5] [25] [26]. This could explain the associations of some outcomes with factors related to intestinal iron absorption, such as prematurity with geophagia and consumption of tannins and fibers, acute fetal distress with antecedent of diabetes in the family. However, other associations are more difficult to explain, such as cesarean section with intestinal parasitosis, geophagia and consumption of tannins and fibers, birth asphyxia (neonatal distress) with previous precocious abortion and consumption of tannins and fibers.

Trials have shown that adverse pregnancy outcomes are in accordance with the severity of maternal iron deficiency anemia [27]. In their systematic review and meta-analysis on hemoglobin concentration and pregnancy outcomes, Sukrat et al. [28] concluded that hemoglobin below $11 \mathrm{~g} / \mathrm{dl}$ increases the risk of preterm birth, low birth weight, and small gestational age in the first trimester and the risk of low birth weight in the third trimester. Lieberman et al. [29] reported an increased odds ratio for preterm birth at $\mathrm{Hb}<7 \mathrm{~g} / \mathrm{dl}$ and hematocrit (Hct) from $41 \%$ to $44 \%$ had lowest risk of premature birth and double at Hct $<$ $37 \%$.

However, Rehema et al. [30] as well as Falahi et al. [31] noted no conclusive evidence that improvement of the iron status during the first and second trimes- 
ter would improve the outcome of pregnancy. In our mind, this might suggest that only the pre-pregnancy status counts, at least dealing with prematurity and LBW. According to the Haider et al.'s review and meta-analysis of 48 randomised trials (17,793 women) and 44 cohort studies (1,851,682 women) [4], randomized trials tend to show a significant association of iron supplements with lower risk of LBW but not on prematurity, while the association involves both in cohort studies. The most concerned periods are the first or second trimesters.

Our study found significant association of GDM with anemia diagnosed at 28 - 32 weeks of gestation, suggesting a potential beneficial effect of iron supplementation if initiated at the beginning of pregnancy. Relationships of iron supplementation and GDM are still controversial. Nutritional iron intake has been found associated with risk of GDM [32]. Some studies found that dietary heme iron intake, but not non-heme iron intake, and supplemental iron intake increased the risk of GDM [33] [34]. Randomized controlled trials in Hong Kong [35] and in Finland [36] found no relationship. Authors who found that the risk was increased observed that it was most increased by high serum iron for some authors [37] [38] [39] [40] or by high ferritin for some others [23] [41] [42] [43]. The debate is still ongoing.

So, even though anemia was found significantly associated with most of complications known to be linked with it, in our series the effect of iron supplementation on pregnancy outcomes is far to be obvious. This could be owed to the fact that improvement of 9.01 - $9.6 \mathrm{~g} / \mathrm{dl}$ observed among anemic women [5] might be too small to induce notable changes in outcomes. Furthermore, Viteri and Berger [44] suggest that maternal hemoglobin between 9.5 and $10.5 \mathrm{~g} / \mathrm{dl}$ in the third trimester correlates with the best clinical outcome.

As we observed it in this study, outcomes found significantly associated with oxidative stress (mostly based on oxidized LDL) seem to overlap those linked to anemic condition. In the present series similarity between complications of anemia and that of oxidative stress is more observed at 28 - 32 weeks of gestation. This strongly suggests that major correction in both anemic and oxidative status should be initiated long before this landmark.

Whether these outcomes are solely related to anemia or oxidative-mediated is difficult to demonstrate. Indeed, irrespective of hemoglobin and iron status, maternal psychosocial or oxidative stress is known to enhance the risk of strictly maternal organic and non organic pathologies (infections, gastritis, hypertensive disorders, insomnia and depression) along with Infant's morbidity (LBW) [45] [46] [47].

\section{Conclusion}

Outcomes found significantly associated with oxidative stress seem to overlap those linked to anemic condition. Similarity between complications of anemia and that of oxidative stress is more observed at 28 - 32 weeks of gestation. This strongly suggests that major correction in both anemic and oxidative status should be initiated long before this landmark. 


\section{Limitation and Strength of the Study}

The main limitation of our study relates to failure of our findings to demonstrate the precise part owed to either anemia or oxidative stress in generation of adverse outcomes observed. The strength of the study comes first from the wide range of outcomes studied in relation to maternal anemia and oxidative stress. Our study also allows to consider 28 - 32 weeks of gestation as an actual landmark for preventive measures against adverse outcomes associated with anemia and oxidative stress.

\section{Author's Contributions}

MMA is the principal investigator, participated in designing the study and was actively involved in data collection and statistical calculations. TUB generated and designed the study. He drafted the first manuscript. MMR participated in designing the study and analyzing the results. All authors contributed in preparing the final manuscript.

\section{Conflicts of Interest}

The authors declare no conflicts of interest regarding the publication of this paper.

\section{References}

[1] Kumar, J.K., Asha, N., Murthy, D.S., Sujatha, M.S. and Manjunath, V.G. (2013) Maternal Anemia in Various Trimesters and Its Effect on Newborn Weight and Maturity: An Observational Study. International Journal of Preventive Medicine, 4, 193-199.

[2] Tandu-Umba, B. and Mbangama, M. (2015) Association of Maternal Anemia with Other Risk Factors in Occurrence of Great Obstetrical Syndromes at University Clinics, Kinshasa, DR Congo. BMC Pregnancy and Childbirth, 15, 183-188. https://doi.org/10.1186/s12884-015-0623-z

[3] Laflamme, E.M. (2012) Maternal Hemoglobin Concentration and Pregnancy Outcome: A Study of the Effects of Elevation in El Alto, Bolivia. McGill Journal of Medicine, 11, 47.

[4] Haider, B.A., Olofin, I., Wang, M., Donna Spiegelman, D., Ezzati, M. and Fawzi, W.W. (2013) Anaemia, Prenatal Iron Use, and Risk of Adverse Pregnancy Outcomes: Systematic Review and Meta-Analysis. British Medical Journal, 346, f3443. https://doi.org/10.1136/bmj.f3443

[5] Mbangama, M.A., Tandu-Umba, B. and Mbungu, M.R. (2019) Routine Iron Supplementation during Pregnancy: Its Reflection on Iron and Oxidative Status in a Cohort of Pregnant Women in Kinshasa, DR Congo. Open Journal of Obstetrics and Gynecology, 9, 98-115. https://doi.org/10.4236/ojog.2019.91011

[6] Schaefer, R.M., Huch, R. and Krafft, A. (2007) Anaemia Working Group. Current Recommendations for the Treatment of Iron Deficiency Anemia. Revue Médicale Suisse, 3, 874-880.

[7] Isler, M., Delibas, N., Guclu, M., Sutcu, R., Bahceci, M. and Kosar, A. (2002) Superoxide Dismutase and Glutathione Peroxidase in Erythrocytes of Patients with Iron Deficiency Anaemia: Effects of Different Treatment Modalities. Croatian Medical 
Journal, 43, 16-19.

[8] Kurtoglu, E., Ugur, A., Baltaci, A.K. and Undar, L. (2003) Effect of Iron Supplementation on Oxidative Stress and Antioxidant Status in Iron Deficiency Anaemia. Biological Trace Element Research, 96, 117-123. https://doi.org/10.1385/BTER:96:1-3:117

[9] Krishnamoorthy, P.M., Natesh Prabhu, R., Mohan, D.M., Sabitha, N., Janakarajan, V.N. and Natesan, B. (2010) Role of Oxidative Stress and Antioxidants in Children with IDA. International Journal of Collaborative Research on Internal Medicine \& Public Health, 2, 2-18.

[10] Akarsu, S., Demir, H., Selek, S. and Oguzoncul, F. (2013) Iron Deficiency Anaemia and Levels of Oxidative Stress Induced by Treatment Modality. Pediatrics International, 55, 289-295. https://doi.org/10.1111/ped.12054

[11] Akca, H., Polat, A. and Koca, C. (2013) Determination of Total Oxidative Stress and Total Antioxidant Capacity before and after the Treatment of Iron-Deficiency Anaemia. Journal of Clinical Laboratory Analysis, 27, 227-230. https://doi.org/10.1002/jcla.21589

[12] El-Shimi, M.S., El-Farrash, R.A., Ismail, E.A., El-Safty, I.A., Nada, A.S., El-Gamel, O.A., Salem, Y.M. and Shoukry, S.M. (2015) Renal Functional and Structural Integrity in Infants with Iron Deficiency Anaemia: Relation to Oxidative Stress and Response to Iron Therapy. Pediatric Nephrology, 30, 1835-1842. https://doi.org/10.1007/s00467-015-3122-6

[13] Knutson, M.D., Walter, P.B., Ames, B.N. and Viteri, F.E. (2000) Both Iron Deficiency and Daily Iron Supplements Increase Lipid Peroxidation in Rats. Journal of Nutrition, 130, 621-628. https://doi.org/10.1093/jn/130.3.621

[14] Srigiridhar, K. and Nair, K.M. (2000) Supplementation with Alpha-Tocopherol or a Combination of Alpha-Tocopherol and Ascorbic Acid Protects the Gastrointestinal Tract of Iron-Deficient Rats against Iron-Induced Oxidative Damage during Iron Repletion. British Journal of Nutrition, 84, 165-173.

[15] Lachilli, B., Hininger, I., Faure, H., Arnaud, J., Richard, M.J., Favier, A. and Roussel, A.M. (2001) Increased Lipid Peroxidation in Pregnant Women after Iron and Vitamin C Supplementation. Biological Trace Element Research, 83, 103-110. https://doi.org/10.1385/BTER:83:2:103

[16] King, S.M., Donangelo, C.M., Knutson, M.D., Walter, P.B., Ames, B.N., Viteri, F.E. and King, J.C. (2008) Daily Supplementation with Iron Increases Lipid Peroxidation in Young Women with Low Iron Stores. Experimental Biology and Medicine, 233, 701-707. https://doi.org/10.3181/0708-RM-233

[17] Joshi, S.R., Mehendale, S.S., Dangat, K.D., Kilari, A.S., Yadav, H.R. and Taralekar, V.S. (2008) High Maternal Plasma Antioxidant Concentrations Associated with Preterm Delivery. Annals of Nutrition and Metabolism, 53, 276-282. https://doi.org/10.4236/ojog.2018.86066

[18] Milman, N., Taylor, C.L., Merkel, J. and Brannon, P.M. (2017) Iron Status in Pregnant Women and Women of Reproductive in Europe. American Journal of Clinical Nutrition, 106,1655S-1662S. https://doi.org/10.3945/ajcn.117.156000

[19] Mbangama, M.A., Tandu-Umba, B. and Mbungu, M.R. (2018) Rationale of a Cohort Study on Risk of Obstetrical Outcomes Associated with Iron Supplementation during Pregnancy. Open Journal of Obstetrics and Gynecology, 8, 598-609. https://doi.org/10.4236/ojog.2018.86066

[20] Mbangama, M.A., Tandu-Umba, B. and Mbungu, M.R. (2018) Baseline Iron Status and Indices of Oxidative Stress in a Cohort of Pregnant Women in Kinshasa, DR 
Congo. Open Journal of Obstetrics and Gynecology, 8, 1476-1486. https://doi.org/10.4236/ojog.2018.814149

[21] HAPO Study Cooperative Research Group (2008) Hyperglycemia and Adverse Pregnancy Outcomes. New England Journal of Medicine, 358, 1991-2002. https://doi.org/10.1056/NEJMoa0707943

[22] Shastri, L., Pammal, R.S., Mani, I., Thomas, T. and Kurpad, A.V. (2016) Oxidative Stress during Early Pregnancy and Birth Outcomes. Public Health Nutrition, 19, 3210-3215. https://doi.org/10.1017/S1368980016001191

[23] Issa, M.M. (2016) Miscarriage and Oxidative Stress of Iron Supplementation during Pregnancy. Global Journal of Medical Research: B Pharma, Drug Discovery, Toxicology \& Medicine, 16, 8-17.

[24] Zhuang, T., Han, H. and Yang, Z. (2014) Iron, Oxidative Stress and Gestational Diabetes. Nutrients, 6, 3968-3980. https://doi.org/10.3390/nu6093968

[25] International Anemia Consultative Group (INACG) (2002) Why Is Iron Important and What to Do about It: A New Perspective. INACG Symposium, Hanoi, 15-16 February 2002, 1-50.

[26] Chandra, I. and Sun, L. (2015) Iron Status and Choice of Iron Therapy during Pregnancy: Advantages and Disadvantages. International Journal of Reproduction, Contraception, Obstetrics and Gynecology, 4, 1264-1271. https://doi.org/10.18203/2320-1770.ijrcog20150695

[27] Scholl, T.O., Hediger, M.L., Fischer, R.L. and Shearer, J.W. (1992) Anemia vs. Iron Deficiency: Increased Risk of Preterm Delivery in a Prospective Study. American Journal of Clinical Nutrition, 55, 985-988. https://doi.org/10.1093/ajcn/55.5.985

[28] Sukrat, B., Wilasrusmee, C., Siribumrungwong, B., McEvoy, M., Okascharoen, C., Attia, J. and Thakkinstian, A. (2013). Hemoglobin Concentration and Pregnancy Outcomes: A Systematic Review and Meta-Analysis. BioMed Research International, 2013, Article ID: 769057. https://doi.org/10.1155/2013/769057

[29] Lieberman, E., Ryan, K.J., Monson, R.R. and Schoenbaum, S.C. (1988) Association of Maternal Hematocrit with Premature Labor. American Journal of Obstetrics and Gynecology, 159,107-114. https://doi.org/10.1016/0002-9378(88)90502-9

[30] Rehema, A., Zilmer, K., Klaar, U., Karro, H., Kullisaar, T. and Zilmer, M. (2004) Ferrous Iron Administration during Pregnancy and Adaptational Oxidative Stress (Pilot Study). Medicina (Kaunas, Lithuania), 40, 547-552.

[31] Falahi, E., Akbari, S., Ebrahimzade, F. and Gargari, B.P. (2011) Impact of Prophylactic Iron Supplementation of Healthy Pregnant Women on Maternal Iron Status and Birth Outcome. Food and Nutrition Bulletin, 32, 213-217. https://doi.org/10.1177/156482651103200305

[32] Helin, A., Kinnunen, T.I., Raitanen, J., Ahonen, S., Virtanen, S.M. and Luoto, R. (2012) Iron Intake, Haemoglobin and Risk of Gestational Diabetes: A Prospective Cohort Study. BMJ Open, 2, pii: e001730. https://doi.org/10.1136/bmjopen-2012-001730

[33] Domellof, M., Thorsdottir, I. and Thorstensen, K. (2013) Health Effects of Different Dietary Iron Intakes: A Systematic Literature Review for the 5th Nordic Nutrition Recommendations. Food \& Nutrition Research, 57, Article ID: 21667. https://doi.org/10.3402/fnr.v57i0.21667

[34] Bowers, K., Yeung, E., Williams, M.A., Qi, L., Tobias, D.K., Hu, F.B. and Zhang, C. (2011) A Prospective Study of Pre-Pregnancy Dietary Iron Intake and Risk for Gestational Diabetes Mellitus. Diabetes Care, 34, 1557-1563. https://doi.org/10.2337/dc11-0134 
[35] Chan, K.K.L., Chan, B.C.P., Lam, K.F., Tam, S. and Lao, T.T. (2009) Iron Supplement in Pregnancy and Development of Gestational Diabetes-A Randomised Placebo-Controlled Trial. BJOG: An International Journal of Obstetrics and Gynaecology, 116, 789-798. https://doi.org/10.1111/j.1471-0528.2008.02014.x

[36] Kinnunen, T.I., Luoto, R., Helin, A. and Hemminki, E. (2014) Supplemental Iron Intake and the Risk of Glucose Intolerance in Pregnancy: Re-Analysis of a Randomised Controlled Trial in Finland. Maternal Child Nutrition, 12, 74-84.

https://doi.org/10.1111/mcn.12139

[37] Behboudi-Gandevani, S., Safary, K., Moghaddam-Banaem, L., Lamyian, M., Goshtasebi, A. and Alian-Moghaddam, N. (2013) The Relationship between Maternal Serum Iron and Zinc Levels and Their Nutritional Intakes in Early Pregnancy with Gestational Diabetes. Biological Trace Element Research, 154, 7-13. https://doi.org/10.1007/s12011-013-9703-y

[38] Akhlaghi, F., Bagheri, S.M. and Rajabi, O. (2012) A Comparative Study of Relationship between Micronutrients and Gestational Diabetes. ISRN Obstetrics and Gynecology, 2012, Article ID: 470419. https://doi.org/10.5402/2012/470419

[39] Al-Saleh, E., Nandakumaran, M., Al-Shammari, M. and Al-Harouny, A. (2004) Maternal-Fetal Status of Copper, Iron, Molybdenum, Selenium and Zinc in Patients with Gestational Diabetes. Journal of Maternal-Fetal and Neonatal Medicine, 16, 15-21. https://doi.org/10.1080/14767050412331283139

[40] Wang, Y., Tan, M., HuanLg, Z., Sheng, L., Ge, Y., Zhang, H., Jiang, M. and Zhang, G. (2002) Elemental Contents in Serum of Pregnant Women with Gestational Diabetes Mellitus. Biological Trace Element Research, 88, 113-118. https://doi.org/10.1385/BTER:88:2:113

[41] Amiri, F.N., Basirat, Z., Omidvar, S., Sharbatdaran, M., Tilaki, K.H. and Pouramir, M. (2013) Comparison of the Serum Iron, Ferritin Levels and Total Iron-Binding Capacity between Pregnant Women with and without Gestational Diabetes. Journal of Natural Science, Biology and Medicine, 4, 302-305.

https://doi.org/10.4103/0976-9668.116977

[42] Soubasi, V., Petridou, S., Sarafidis, K., Tsantali, C., Diamanti, E., Buonocore, G. and Drossou-Agakidou, V. (2010) Association of Increased Maternal Ferritin Levels with Gestational Diabetes and Intra-Uterine Growth Retardation. Diabetes \& $\mathrm{Me}$ tabolism, 36, 58-63. https://doi.org/10.1016/j.diabet.2009.06.010

[43] Chen, X., Scholl, T.O. and Stein, T.P. (2006) Association of Elevated Serum Ferritin Levels and the Risk of Gestational Diabetes Mellitus in Pregnant Women: The Camden Study. Diabetes Care, 29, 1077-1082. https://doi.org/10.2337/dc06-0164

[44] Viteri, F.E. and Berger, J. (2005) Importance of Pre-Pregnancy and Pregnancy Iron Status: Can Long-Term Weekly Preventive Iron and Folic Acid Supplementation Achieve Desirable and Safe Status? Nutrition Reviews, 63, S65-S76. https://doi.org/10.1301/nr.2005.dec.S65-S76

[45] Tandu-Umba, B., Dedetemo, K.D. and Mananga, L.G. (2014) Maternal Stress and Pregnancy Outcomes. Open Journal Of Obstetrics and Gynecology, 4, 361-370. https://doi.org/10.4236/ojog.2014.47054

[46] Lilliecreutz, C., Larén, J., Sydsj, G. and Josefsson, A. (2016) Effect of Maternal Stress during Pregnancy on the Risk for Preterm Birth. BMC Pregnancy Childbirth, 16, 5. https://doi.org/10.1186/s12884-015-0775-x

[47] Khanna, A., Dubey, M. and Sinha, R. (2010) Oxidative Stress and Its Correlation with the Severity of Maternal Anemia. Indian Journal of Preventive and Social Medicine, 41, 231-236. 